\title{
Neural graphs: small-worlds, after all?
}

\author{
Michelle Rudolph-Lilith*, Lyle E Muller \\ From The Twenty Third Annual Computational Neuroscience Meeting: CNS*2014 \\ Québec City, Canada. 26-31 July 2014
}

In recent years, small-world graphs have gained considerable interest as models of real-world systems, which often display features residing between regularity and randomness. The most notable of these models is the Watts-Strogatz graph [1], though alternatives have been proposed [2]. The unifying characteristics of these models are that any two nodes are joined with a small number of links between them (i.e. short path length), while at the same time connected node pairs exhibit an abundance of triangular relations resulting in a high degree of local redundancy (i.e. high clustering).

Theoretical investigations of small-world graph models have generally applied asymptotic evaluations in the limit of large system size [3] or the continuum approximation [4] to the algorithmic definition of the graph, in the absence of an analytic representation. In this study, we introduce a generative model of directed small-world graphs, a canonical model of Watts-Strogatz digraphs, and propose an approach that yields the graph's defining adjacency matrix in algebraic terms, with the goal to provide mathematically rigorous access to the study of finite-size small-world graphs [5]. The proposed approach makes use of random annihilation operators whose algebraic properties can be utilized to assess algebraically well-defined graph-theoretic measures in an analytically exact framework, valid nonasymptotically for all graph sizes. We demonstrated the application of our approach by calculating, for the first time, the asymmetry index and total clustering coefficient of small worlds in an exact fashion.

We then utilize the exact nonasymptotic expression for the clustering coefficient in order to assess the small-worldness of structural brain networks in an analytic setting. Using the number of nodes and edges of the given brain networks to construct the equivalent small-world network, we observe that a significant edge rewiring of at least $20 \%$ up to $60 \%$ is required to produce the small-worldness

\footnotetext{
* Correspondence: rudolph@inaf.cnrs-gif.fr

Unité des Neurosciences, Information et Complexité (UNIC), CNRS UPR-3293,
} Gif-sur-Yvette, 91198, France

Submit your next manuscript to BioMed Central and take full advantage of:

- Convenient online submission

- Thorough peer review

- No space constraints or color figure charges

- Immediate publication on acceptance

- Inclusion in PubMed, CAS, Scopus and Google Scholar

- Research which is freely available for redistribution Submit your manuscript at
www.biomedcentral.com/submit C BioMed Central 